\title{
Project OSMOSE: implementation and first results of voltage regulation tests from wind power plants
}

\author{
Alessio Siviero \\ Innovation Factory System Operator \\ Terna S.p.A. \\ Rome, Italy \\ alessio.siviero@terna.it
}

\author{
Leonardo Petrocchi \\ Innovation Factory System Operator \\ Terna S.p.A. \\ Rome, Italy \\ leonardo.petrocchi@terna.it
}

\author{
Alessandro Arienti \\ Edison $\operatorname{SpA}$ \\ Milan, Italy \\ alessandro.arienti@edison.it
}

\author{
Fabia Gasparotto \\ Innovation Factory System Operator \\ Terna S.p.A \\ Rome, Italy \\ fabia.gasparotto@terna.it \\ Giacomo Petretto \\ Innovation \\ Enel Global Power Generation \\ Pisa, Italy \\ giacomo.petretto@enel.it \\ Pasquale Morelli \\ Edison SpA \\ Milan, Italy \\ pasquale.morelli@edison.it
}

\author{
Luca Orrù \\ Innovation Factory System Operator \\ Terna S.p.A. \\ Rome, Italy \\ luca.orru@terna.it \\ Massimo Mannelli \\ Innovation \\ Enel Global Power Generation \\ Rome, Italy \\ massimo.mannelli@enel.it
}

\begin{abstract}
The large scale diffusion of variable Renewable Energy sources (vRES) and the simultaneous dismission of conventional power plants, while being recognized fundamental for the transition to a fully decarbonized energy system, pose new challenges to electricity systems, also in terms of stability of the main grid parameters (frequency and voltage) due to the decrease of the number of rotating masses connected to the grid. This context determines the need to expand the group of resources which can offer voltage and frequency regulation services. In the premise of the Horizon 2020 OSMOSE project Work Package 5, technical characterization of $v R E S$ resources providing voltage regulation is investigated by involving two wind power plants (one of which integrates a storage systems) located in Basilicata. In addition to retracing the voltage regulation typical requirements and the implementations realized on the plants in order to allow the service provision, this paper means to offer a first glance at the abovementioned experimentation and first results. Keywordsflexibility, renewable energy sources, voltage regulation.
\end{abstract}

\section{INTRODUCTION}

The OSMOSE (OPTIMAL SYSTEM-MIX OF FLEXIBILITY SOLUTIONS FOR EUROPEAN ELECTRICITY) project is a H2020 EU funded project that aims to foster the integration of new flexibility resources which can be used for a better integration of RES capacity that is steeply increasing due to the Energy Transition. The approach chosen is global as it considers at the same time the increased need of flexibilities in the system (mainly improved balance of supply and demand in electricity markets, provision of existing and future system services and allowance of a dynamic control of electricity flows) and the sources of flexibilities (RES, demand-response, grid and new storages). In order to prove the viability of the proposed approach in real operating conditions, four demonstrators have been included in the project, leveraging on the cooperation of four European Transmission System Operators: RTE (France); REE (Spain), Eles (Slovenia) and Terna (Italy).

The italian demo aims to demonstrate multiple grid services based on Renewable Energy Sources (RES), Demand-Side Response (DSR) and Dynamic Thermal Rating
(DTR) devices, some of which coordinated through a smart management system. The demonstrator area was selected by identifying those italian grid portions in which the testing of flexibility services provision as well as the application of congestion management resources would have been the most effective. This area was identified as a $150 \mathrm{kV}$ portion between Apulia and Basilicata regions (Figure1). The demonstrator is therefore composed of:

- 7 main $150 \mathrm{kV}$ lines as the test field

- 2 Wind power plants

- 7 industrial loads (not discussed in this paper)

The partner engagement in the Italian demo involves the whole value chain: Utilities (TERNA, Edison Rinnovabili (formerly E2i Energie Speciali), ENEL GREEN POWER), R\&D center and universities (ENSIEL, RSE), industrial providers (ABB, IBM, ENGINEERING) and aggregators (ENEL X, COMPENDIA, EDISON).

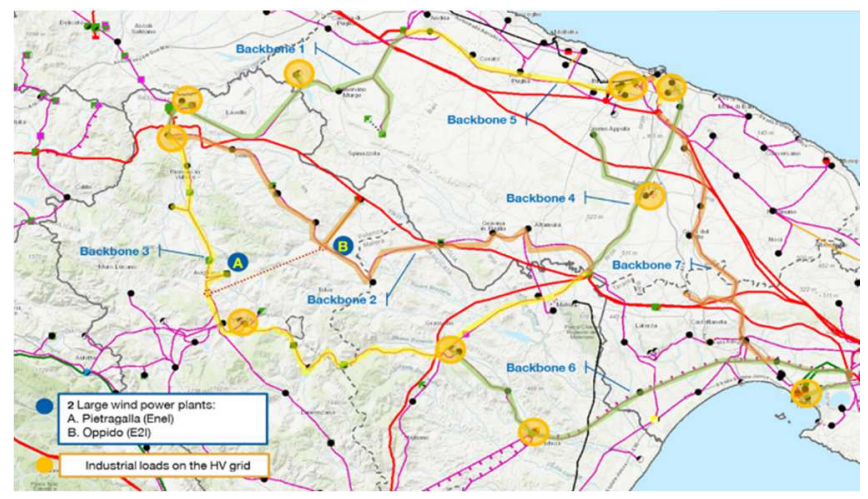

Figure 1. Demonstrator area and flexibilities resources

This paper describes the main activities performed until now regarding the voltage regulation provision implementation and testing from the two wind power plants involved in 
OSMOSE, after having provided a needed introductory part on voltage regulation requirements.

The demonstration activities started in May 2021 and will take place for approximately 10 months duration. Complete test results will therefore be available by the end of 2021 .

\section{Voltage REgUlation}

Voltage regulation is the group of activities needed to keep voltage fluctuations on the electricity transmission nodes between fixed limits. Since voltage at the nodes of the electricity transmission grid is also determined by reactive power transfers occurring over the lines that constitute the grid, the variation of production or absorption of reactive power is the main instrument utilized for voltage regulation (for instance, this is traditionally done by acting on the alternator's excitation current) [1].

Conventional thermoelectric plants are contributors to voltage regulation: production groups, transformers, power factor correction systems are the plant components usually involved in such regulation. Furthermore, users directly connected to the transmission grid are indirectly asked to contribute to voltage regulation by adopting actions in order to keep the power factor inside a fixed interval for every possible load condition [2].

However, the dismission of conventional plants, in the complex frame of the energy transition, poses the threat of a reduction in the reactive power regulation capacity for the Electricity System. [3] Consequently, also other energy sources which were never directly adopted for voltage regulation started being investigated in this sense.

Indeed, the Requirement for Generators (RfG) code established in 2016 that both new and existing energy production plants need to be technically capable of providing voltage regulation; in particular, the existing plants, both programmable and non-programmable, which were not obliged to voltage regulation provision before, need to be technically updated to guarantee voltage regulation provision, (exceptions are however foreseen). Accordingly, in the perimeter defined by the Delibera 300/2017/R/eel from the Italian regulator, Terna is defining a pilot project aimed at promoting voltage regulation by those plants, both programmable and non-programmable, which need specific technical updates in order to be ready for voltage regulation (the pilot project draft requirements have been under consultation for the past months) [4].

Focusing on wind power plants, the Italian Grid Code prescribes the main functionalities that are required to the plant in terms of voltage regulation [5]. The plant in parallel to the power grid is required to participate to the Electric System voltage control. Such control must be performed referring to the voltage signal measured at the HV side of the plant. Terna communicates the reference voltage signal to the plant either following a local logic (Terna communicates the reference value and the plant applies it) or a remote logic (Terna communicates a variable signal from its Remote Control Centre through a direct communication and the plant directly follows). In the following, more details are given about these two types of regulation according to the most recent update of the Italian Grid Code [5].

\section{A. Local regulation (Vref set-point)}

Reactive power insertion or absorption must be provided accordingly to a characteristic $\mathrm{Q}=\mathrm{f}(\Delta \mathrm{V})$ as the one represented in Figure 2 - reactive power provision must be proportional to the difference between the set-point and the HV measured voltage value $(\Delta \mathrm{V})$. Terna will communicate a reference $\mathrm{HV}$ voltage set-point in a range between the $95 \%$ and $105 \%$ of the plant's nominal voltage and the plant regulator will follow. According to the curve in Figure 2, two linear traits are implemented in the inductive and capacitive regions as a function of the percentual $\Delta \mathrm{V}$; a dead band might also be included in the curve. Outside of the $\Delta \mathrm{V}_{\min }$ and $\Delta \mathrm{V}_{\max }$ bands the plant must provide its maximum power reaction contribution (i.e., in general, the one corresponding to the $35 \%$ available active power).

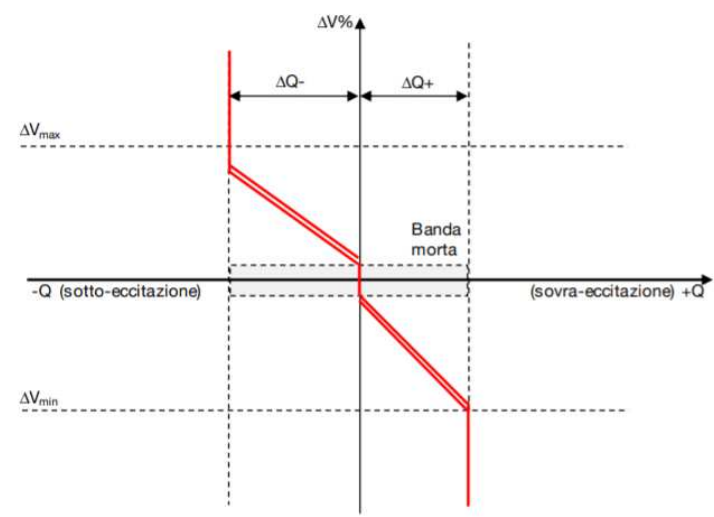

Figure 2. Characteristic $\mathrm{Q}=\mathrm{f}(\Delta \mathrm{V})$ curve

Following a $\Delta \mathrm{V}$ event, the plant is requested to provide the $90 \%$ of the required reactive power requested in $2 \mathrm{~s}$ and the $100 \%$ of the required $\mathrm{Q}$ in $5 \mathrm{~s}$ with a precision lower than $5 \%$ of the maximum available reactive power.

\section{B. Centralized regulation (Q set-point)}

The plant must be equipped to receive a reactive power set-point from Terna; the setpoint is elaborated by a remote system and exchanged through a proper telecommunication system. The plant needs to follow this set-point up to the plant capability limits and with a precision not lower than $5 \%$ of the maximum available reactive power. The plant also communicates the maximum available reactive power limits in real time, at least every $4 \mathrm{~s}$, in order to allow Terna an accurate and proper calculation of the set-point. Following a Q set-point, the plant is requested to provide the $90 \%$ of the required reactive power reaction in $2 \mathrm{~s}$ and the $100 \%$ of the required $\mathrm{Q}$ in $5 \mathrm{~s}$ with a precision lower than $5 \%$ of the maximum available reactive power.

\section{PLANTS INVOLVED AND IMPLEMENTATION}

As specified in the Introduction, part of the OSMOSE project experimentation relates to innovative services provided by RES plants. Of the plants involved, one is owned by Enel Green Power (EGP) and located in Pietragalla and two are owned by Edison Rinnovabili (formerly E2i) and 
located in Vaglio (both of them, "Vaglio" and "Vaglio IR" refer to the same point of connection, ).

The Pietragalla EGP plant is an integrated RES + Storage power plant and is made of a $18 \mathrm{MW}$ wind power plant $(9 \times 2$ MW turbines) and a 2MW/MWh Battery Energy Storage System. The E2i power plants considered are the "Vaglio" and "Vaglio IR" wind farms for a total $35 \mathrm{MW}$ power (20MW + $15 \mathrm{MW}$ plant, $14 * 2.5 \mathrm{MW}$ turbines); from now on they will be referred to uniquely as "Vaglio" power plant. The core of the experimentation is to test automatic voltage regulation (AVC) and Synthetic Inertia. Since the focus of this paper is voltage regulation, a focus on the implementations performed on the plants in Osmose to provide the service is given.

\section{A. Pietragalla}

The implementations carried out in the Pietragalla plant are listed as follows:

- The Master Scada of the Pietragalla plant has been updated to implement the regulation algorithms and control logics that allow for voltage regulation provision. Thanks to these developments, the Master Scada has now available four "regulation modes" through which local and centralized regulation can be either directly managed from the Master Scada (e.g., the Q or V set-points are sent locally from the Master Scada HMI or from the EGP Control Room) or from the TSO (in this case, the set-points are sent from the TSO to the EGP Control Room, acting as a gateway, which then redirects them to the Pietragalla Master Scada). New functionalities were also added in order to guarantee for the correct coordination between the wind power plant and storage system during voltage regulation service provision.

The communication chain between plant and Terna has been updated so to allow for the service provision. In particular, a considerable amount of work has regarded the definition and implementation of the most appropriate list of set-points, signals, measurements and commands that would guarantee the service provision most efficiently.

The data exchange between EGP local control room and Terna is carried out using the IEC 60870-5-104 protocol and allows to send Q or V set points from the TSO control room to the plant, choose the regulation mode, and receive $\mathrm{Q}$ and $\mathrm{V}$ local measurements, useful data (e.g., the real plant Q capability) and feedback from the plant.

An overall view of the asset architecture after the implementations is given in Figure 3 .

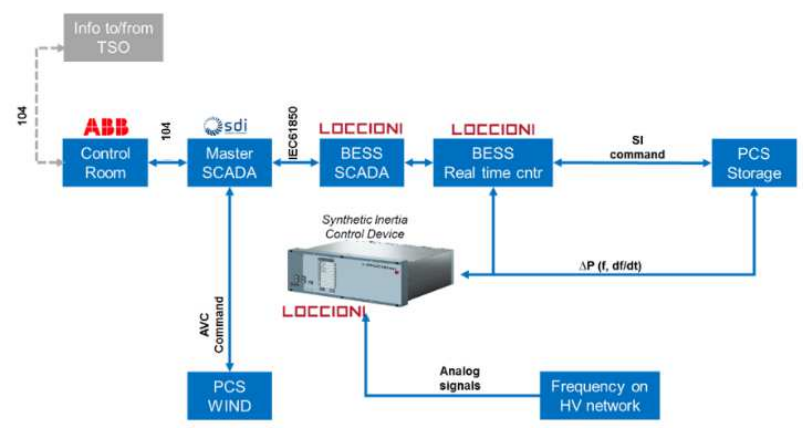

Figure 3. Pietragalla Plant control architecture

\section{B. Vaglio}

The implementations carried out in the Vaglio plant are listed as follows:

- The experimental activities have required a preliminary design and a modification of the communication architecture between Terna control room and the local wind farm control embedded equipment. An additional software module has been added on the local equipment to allow the exchange of data, set-points and feedback in the loop regulation for both voltage and reactive power regulation. More specifically, the power plant controller (PPC) has been updated to include the control and regulation algorithms necessary for AVC provision: indeed, the PPC is deputed (i) to receive and transmit data information towards the center (TSO control room through a gateway TSO Data Concentrator) and (ii) to send reactive power set-points to the Power Control Unit Gamesa (PCU) which is connected to all the wind turbines (Vaglio and Vaglio IR wind farms). When remote regulation is on, the PPC either just passes the Q setpoint to the Power Control Unit or calculates the Q set-point for the plant, starting from the received V set-point, and then sends it to the PCU.

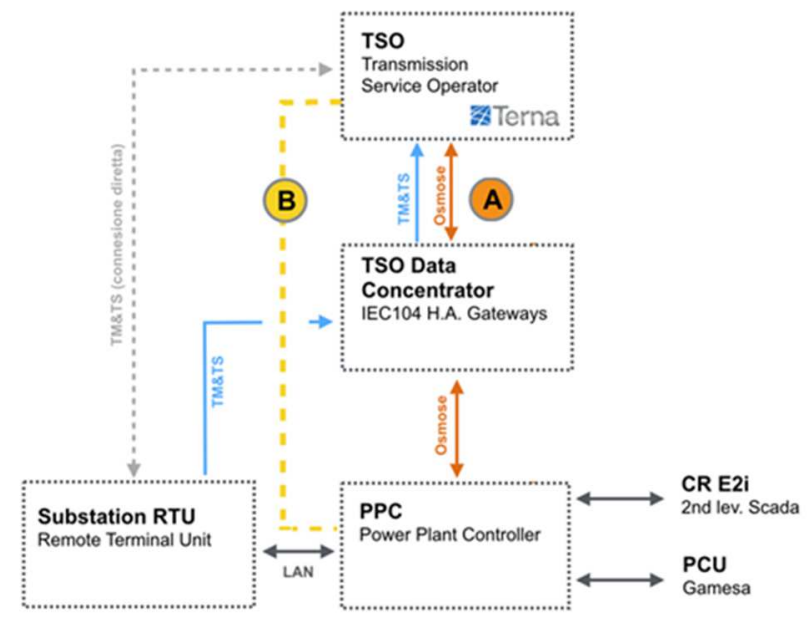

Figure 4. Local power plant controller (PPC) monitoring and communications link architecture

During the implementation phase, analogously to what has been done with the Pietragalla plant, a communication chain between the plant and the TSO control room has been 'revamped', in order to allow for the remote control of the AVC regulation. More specifically, the local PPC now communicates with Terna control room through a gateway concentrator realized both (i) for monitoring Vaglio electrical substation as the Italian Grid Code regulation requires and (ii) for exchanging an additional list of parameters especially designed for OSMOSE experimental purposes. An overall view of the architecture is given in Figure 4. As established by the Italian Grid Code the data exchange is carried out with the IEC 60870-5-104 protocol. Date exchange parameters were chosen and implemented analogously to what done in Pietragalla. 


\section{EXPERIMENTAL DATA SETS}

After EGP and Edison Rinnovabili conduct locally their own commissioning tests for the implemented systems, to verify their correct implementation and functioning, a communication test is organized with Terna's support and the data exchange correctness between TSO and plant is tested.

After that, the experimentation was set to begin with local tests i.e., the set-point generation is commanded by the local control systems following test specifications elaborated with Terna's support. After first evidence was collected during local tests and eventual corrections have been implemented on the systems, remote tests are being performed i.e., with setpoints sent by the TSO control room. This allows testing the whole technology chain performance and to appreciate the differences introduced by the communication chain between plant and TSO.

Test specifications and experimentation results reported hereinafter refer to the Vaglio plant, which was the first to complete the technical implementations needed for the experimentation set-off.

\section{A. Test specifications}

Starting from the technical specifics contained in the technical specifications from Terna's pilot project [6], various test specifications were elaborated for the local and remote tests to be performed with the Vaglio and Pietragalla plant.

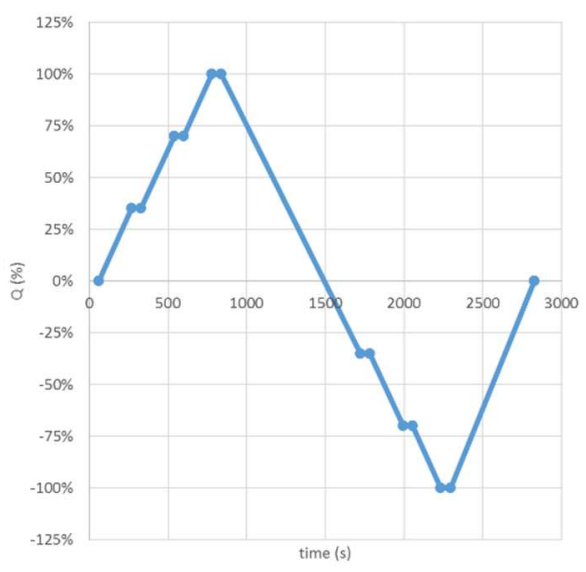

Figure 5. Reference Q set-point test ("static" regulation)

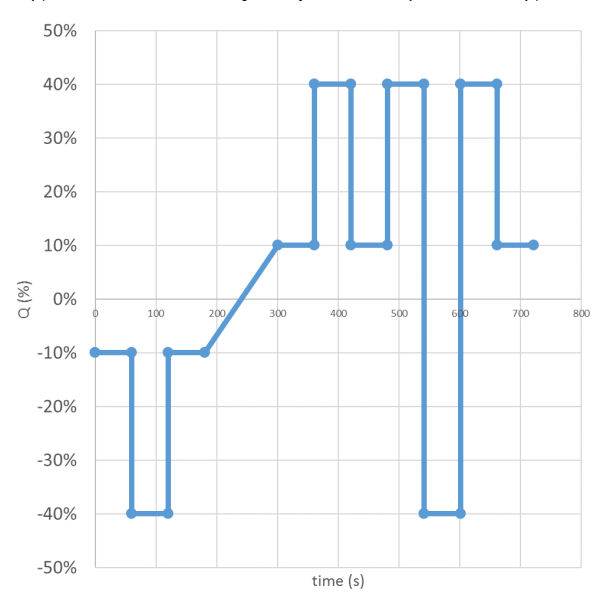

Figure 6. Reference Q set-point test (“dynamic” regulation)
The graph shown in Figure 5 depicts a slow variation $(10 \% / \mathrm{min})$ of the reactive power set-point ("test $\# 1$ " in the following): the main purpose of this test typology is to assess the P-Q capability of the plant to reach the demanded set-point and its precision. The reactive power set-point is not an absolute value but a percentage given by the ratio of the desired reactive power value and the available Q capability. So, for instance, when the plant is asked for a $100 \%$ set-point it has to provide the maximum available Q. In the proposed test, the plant is asked to linearly increase its Q provision from $0 \%$ to $35 \%$ to $70 \%$ and to $100 \%$ and then decrease, following a similar path but contemplating also a sign change in $\mathrm{Q}$. The linear part of the curve has a $10 \% / \mathrm{min}$ slope - during local tests in Vaglio, such slope has been reproduced by sending 30s apart set-points. On the other hand, when one of the "main" set-points (for instance: $35 \%, 70 \%, 100 \%$, in the first rising part of Figure 5) is sent the controller waits 60 s before sending the next one.

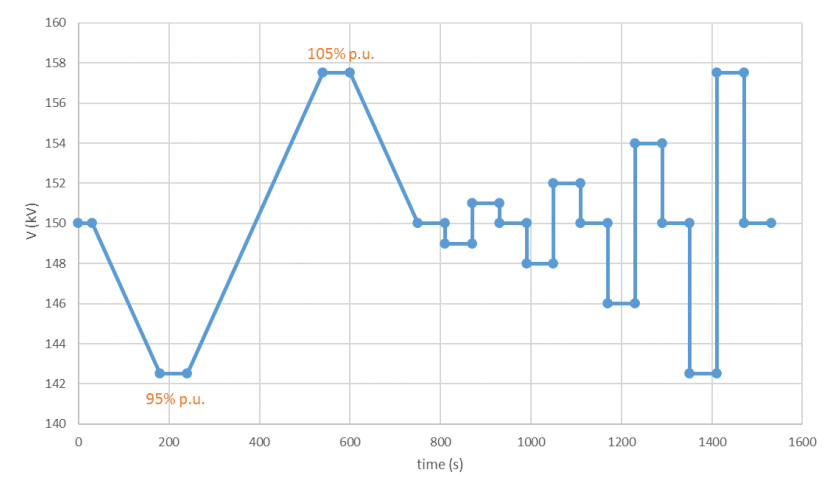

Figure 7. Reference V set-point test

The second test ("test \#2" in the following), shown in Figure 6, depicts step variations of the reactive power-set point: the scope is to test and evaluate the dynamic response of the plant. Step variations are asked to the plant controller both in the inductive and capacitive regions of the capability, and also a sign change of the Q set-point is contemplated, from a $40 \%$ to $-40 \%$ Q level.

The third test shown in Figure 7 ("test \#3" in the following) depicts a reference $\mathrm{V}$ set point variation: analogously to what seen for the other two test typologies both static and dynamic responses are analyzed, but in one single session, while ideally asking the plant to modify the HV voltage between $95 \%$ and $105 \%$ of the nominal value. In this case, the setpoint sent is the $\mathrm{HV}$ voltage real desired value. In the first part of the test the plant is asked to slowly vary the HV voltage, by sending set-points with a $2 \% / \mathrm{min}$ slope and reaching both the $95 \%$ and $105 \%$ limit. The plant is then asked to respond to some increasing step set-points. When a V set-point is sent, the plant Master Scada calculates the $\mathrm{Q}$ value according to the implemented $\mathrm{Q}=\mathrm{f}(\Delta \mathrm{V})$ curve as in Figure 2. With the purpose of assessing the impact of the $\mathrm{Q}=\mathrm{f}(\Delta \mathrm{V})$ slope on the regulation and actually see if the plant follows and how, three different $\mathrm{Q}=\mathrm{f}(\Delta \mathrm{V})$ curves were tested for the Vaglio plant. 
The values describing this curve are reported in the Table

\begin{tabular}{l|c|c|c|c|c} 
& $\begin{array}{c}\text { Dead band } \\
(\mathrm{kV})\end{array}$ & $\begin{array}{c}\Delta \mathrm{Vmax} \\
(\mathrm{kV})\end{array}$ & $\begin{array}{c}\Delta \mathrm{Vmin} \\
(\mathrm{kV})\end{array}$ & $\begin{array}{c}\Delta \mathrm{Q}+ \\
(\mathrm{Mvar})\end{array}$ & $\begin{array}{c}\Delta \mathrm{Q}- \\
(\mathrm{Mvar})\end{array}$ \\
\hline $\begin{array}{l}\text { Curve \#1 } \\
\text { ("slow") }\end{array}$ & \pm 1 & 5 & -5 & 11 & -18 \\
\hline $\begin{array}{l}\text { Curve \#2 } \\
\text { ("medium") }\end{array}$ & \pm 1 & 3 & -3 & 11 & -18 \\
\hline $\begin{array}{l}\text { Curve \#3 } \\
\text { ("fast") }\end{array}$ & \pm 0.5 & 1,5 & $-1,5$ & 11 & -18
\end{tabular}

Table 1. $\mathrm{Q}=\mathrm{f}(\Delta \mathrm{V})$ curves parameters

\section{B. Preliminary test results}

Qualitative results for the conducted tests are shown in the following along with some considerations. More thorough analyses are to be performed in the remainder of the demonstration.

During one of the experimental sessions for test \#1, the active power oscillated between approximately 8 and $17 \mathrm{MW}$, meaning that almost the whole capability should have been available for provision (see the P-Q capability curve for comparison, Figure 8).

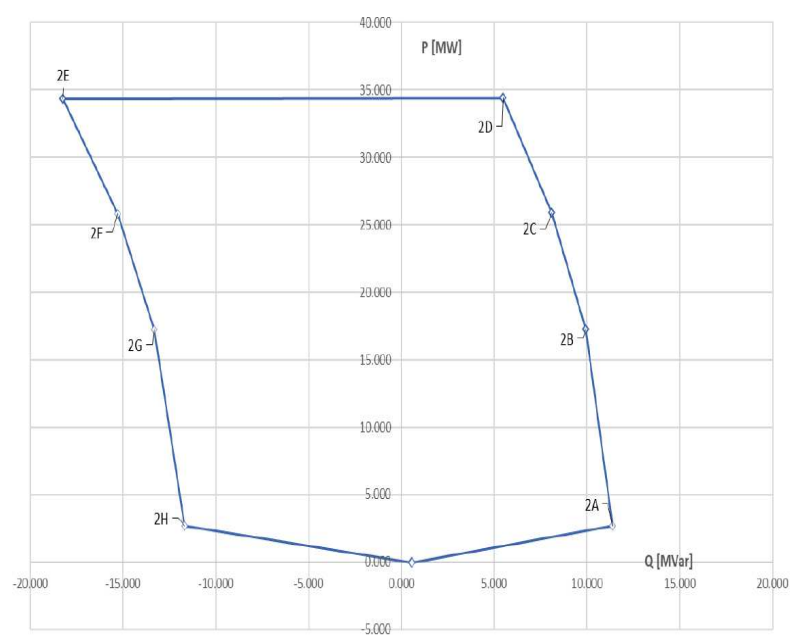

Figure 8. Vaglio plant P-Q capability at the point of connection

The results for one of the experimental sessions for test \#1 are reported in Figure 9. The graph in Figure 9 represents the $Q$ value (which reflects a percentage of the available real capability) versus the $\mathrm{Q}$ delivered by the plant. At a first glance, it is possible to notice how the system does not deliver the requested $\mathrm{Q}$ above or under a certain value (about \pm 7Mvar) showing a sort of saturation issue to be better analysed. For the remaining set-points, the requested reactive power is reached; an analysis on the times needed to reach each of the set-points (considering all the set-points, including the 30 s ones) reveals that it takes about 8-11 s (in few cases, more than $20 \mathrm{~s}$ ) to reach the $90 \%$ of the requested value (the Grid Code requires $2 \mathrm{~s}$ ). More thorough analysis which will

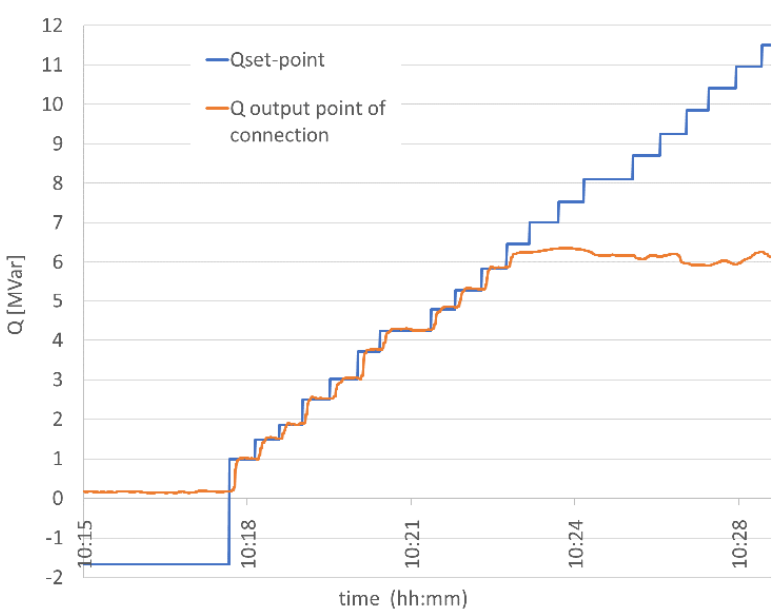

Figure 9. Excerpt from Q set-point test ("static" regulation)

be performed when the experimentation is complete will follow.

The active power for the experimental session \#2 oscillates between about 12 and $14 \mathrm{MW}$; the test output can be observed in Figure 10. Similar features to those observed for test \#1 can be traced here but in this case no saturation is shown since the reactive power requested does not reach that level. What seems to appear clearer from this test is a strong dependency between the reactive power "jump" requested and the time needed to reach it. The $\mathrm{Q}$ requests bringing the set-point from about 1.2 Mvar to about 5 Mvar are fully satisfied in about 20 $\mathrm{s}$, while the $\mathrm{Q}$ request from 5 Mvar to about -4 Mvar needs more than $40 \mathrm{~s}$ to be satisfied. Also the presence of ripples in

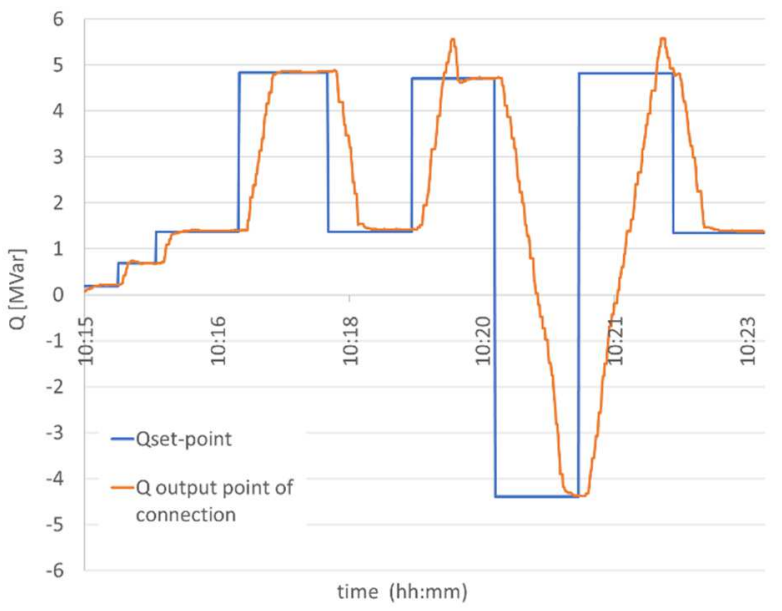

Figure 10. Q set-point test (“dynamic” regulation)

the curve needs further investigation.

For test \#3, results are shown in Figure 11. The test was repeated adopting different underlying $\mathrm{Q}=\mathrm{f}(\Delta \mathrm{V})$ curves - these $\mathrm{Q}=\mathrm{f}(\Delta \mathrm{V})$ curves implemented in the plant control system allow for a different $\mathrm{Q}$ response when a certain $\mathrm{V}$ set-point is sent. This can be appreciated in the test results obtained. For instance, for the "fastest" parametrization test the plant is correctly asked to immediately deliver the maximum possible reactive power, meaning that for a voltage difference less than $1.5 \mathrm{kV}$ the power plant is asked to provide almost its full 


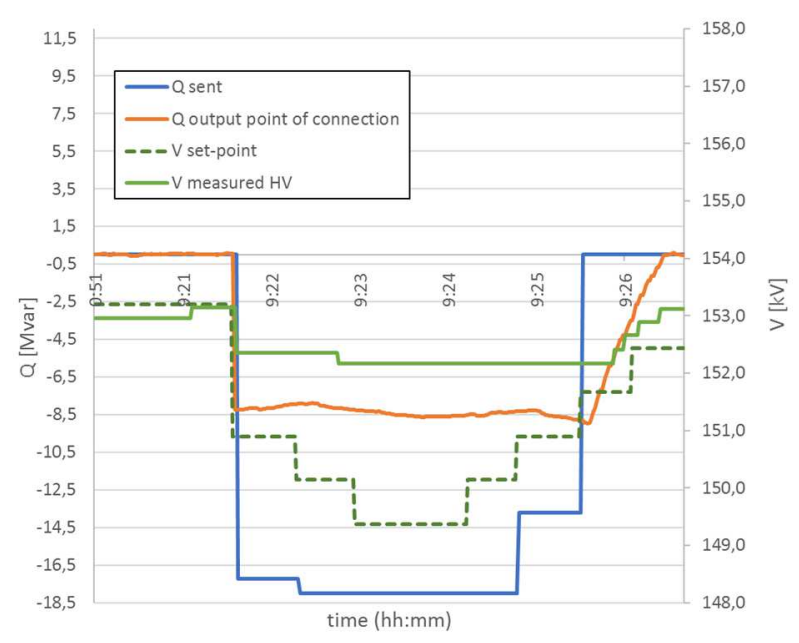

Figure 11. Excerpt from V set-point test

capability. Voltage variation obviously follows the reactive power variation. Analogously to the tests performed with the $\mathrm{Q}$ set-points, also in this case the plant cannot provide the requested level above or below a certain requested $\mathrm{Q}$ level. Times needed to reach the set-points are around $30 \mathrm{~s}$ or more, with the longest times registered when a sign change in reactive power happens.

\section{CONCLUSIONS AND NEXT STEPS}

The OSMOSE project aims at exploring new sources of flexibilities for the grid. In this frame, experimentation on services like voltage regulation by RES plants, which were not required before to actively regulate reactive power, is aimed at providing the System with a new source of flexibility. The modifications and upgrades on the plants' control system needed to allow for voltage regulation provision were based on the Italian Grid Code requirements and brought to the implementation of both "local" and "centralized" regulations, making the plants available to vary reactive power following a reference voltage or reactive power set-points. Tests were then conceived in order to test the static and dynamic response of the implemented solutions. The first local test sessions in Vaglio revealed the wind plant potential in providing a reactive power response - from preliminary analyses it was observed the plant's capability in reaching the desired setpoints, but typically with higher times than expected (more than what required by the Grid Code) and depending on the reactive power delta required. Further analysis on the data will give more inputs on the accuracy of the performance and on its dynamics. Tests will follow in Pietragalla where an electrochemical storage system is installed, providing also a technology comparison. Remote tests will finally allow to test the full chain performance and provide comprehensive information about voltage regulation from RES plants.

\section{ACKNOWLEDGMENT}

The present paper is realized by the members of the OSMOSE consortium, funded by the H2020 programme under the call: LCE4-2017- Low carbon electricity (project ID 773406).

\section{REFERENCES}

[1] Terna, Allegato A.14 Codice di Rete - Partecipazione alla regolazione di tensione.

[2] Terna, Allegato A.16 Codice di Rete - Sistema automatico per la regolazione della tensione (SART) per centrali elettriche di produzione.

[3] Terna., Contesto ed evoluzione del Sistema Elettrico., 2019.

[4] Terna, Regolamento recante le modalità per la partecipazione alla fornitura del servizio di regolazione di tensione tramite impianti connessi alla RTN a seguito di adeguamenti impiantistici., 2020.

[5] Terna, Allegato A.17 del Codice di Rete - CENTRALI EOLICHE Criteri generali di connessione alle reti AT - Sistemi di protezione regolazione e controllo.

[6] Terna, Definizione dei requisiti tecnici per la partecipazione al servizio di regolazione gerarchica di tensione tramite impianti programmabili e non programmabili, connessi alla RTN a seguito di adeguamenti impiantistici, 2020. 